\title{
IMPACTO DE UN MODELO INTEGRADO PARA EL USO RACIONAL DE ANTIMICROBIANOS (PROYECTO MIURA) EN UN ÁREA DE SALUD (*)
}

\author{
Javier Colomina Rodríguez (1,2), Victoria Domínguez Márquez (1,2), Flor Gimeno Vilarrasa (2), \\ Gema Sarrió Montes (3) y Antonio Guerrero Espejo (2). \\ (1) Servicio de Microbiología Hospital Universitario de La Ribera. Alzira. Valencia. \\ (2) Unidad de Investigación en Patología Infecciosa. Hospital Universitario de La Ribera. Alzira. Valencia. \\ (3) Farmacia Hospitalaria. Hospital Universitario de La Ribera. Alzira. Valencia.
}

\section{RESUMEN}

Fundamento: MIURA (Modelo Integrado para el Uso Racional de Antimicrobianos) es un proyecto que se inicio en enero de $2004 \mathrm{y}$ finalizó en diciembre de 2006. Mediante intervenciones trimestrales y formativas dirigidas a médicos, farmacéuticos y pacientes, pretendió mejorar el uso de antibióticos en un área de salud. El objetivo ha sido analizar la evolución en el consumo de antibióticos y evaluar la repercusión de la implantación de este proyecto en un área de salud.

Método: Se realizó un estudio comparativo sobre la evolución de la dispensación de antibióticos con receta durante los periodos 2000-03 (pre-MIURA), 2004-06 (MIURA) y 2007-08 (post-MIURA) en el Departamento de Salud 11 de la Comunidad Valenciana. La información se obtuvo a través de la aplicación informática GAIA (Generalitat Valenciana) que recoge información sobre los medicamentos facturados por las oficinas de farmacia a través de las recetas oficiales. Como unidad técnica de medida se empleó la DHD (Dosis diaria definida/1.000 Habitantes/Día).

Resultados: Durante el periodo de implantación del proyecto (2004 a 2006), se detectó un descenso global de 4,02 DHD en el consumo de antibióticos, lo que representa una reducción global y significativa del $15 \%(\mathrm{p}<0.05)$. Durante el periodo del MIURA se observó una disminución estadísticamente significativa en los valores de DHD para los grupos terapéuticos de macrólidos (especialmente claritromicina) y de cefalosporinas; también se detectó una disminución, aunque no significativa, para el grupo de las quinolonas, mientras que los grupos correspondientes a las penicilinas y a otros antibióticos no presentaron cambios. En el periodo post-MIURA se detectó un nuevo incremento en la dispensación de los antibióticos.

Conclusiones: El programa MIURA ha influido positivamente en la disminución de la prescripción de antibióticos en nuestra área de salud, como demuestra el descenso en DHD durante el periodo de intervenciones.

Palabras clave: Antibióticos. Uso fuera de lo indicado. Utilización de medicamentos.

Correspondencia:

Javier Colomina.

Servicio de Microbiología

Hospital Universitario de La Ribera

Carretera de Alzira-Corbera, Km 1. 46600-Alzira (Valencia).

jcolomina@hospital-ribera.com
ABSTRACT

\section{Impact of Integrated Model for Rational Use of Antibiotics in a health area (project MIURA)}

Background: MIURA (Integrated Model for the Rational Use of Antimicrobials) is a project that began in January 2004 and ended in December 2006. Through quarterly and training interventions intented for physicians, pharmacists and patients, pretends to improve antibiotic use in a health area. The goal was to analyze the evolution of antibiotic consumption and assess the impact of implementing this project in a health area.

Method: A comparative study on the evolution of antibiotic dispensed during the periods 2000-03 (pre-MIURA), 2004-06 (MIURA) and 2007-08 (post-MIURA) in the Department of Health 11 of Valencia was conduced. Antibiotics information was obtained through the GAIA application (Generalitat Valenciana) that collects information about the drugs billed by the pharmacy through the official prescriptions. Technical unit of measurement was used DHD (daily dose defined/1.000 inhabitants / day).

Results: During the implementation of the project (2004-2006), antibiotic consumption was reduced in 4.02 DHD. It represents a statistically significant overall reduction from $15 \%(p=<0.05)$. Since interventions started, a statistically significant decrease in DHD values was observed for macrolides (especially clarithromycin) and cephalosporines. It also exist an average decrease, not significant, for the group of quinolones, whereas penicillins and other antibiotics haven't showed variability in the consumption data. In the post-MIURA period was detected a further increase in the dispensing of antibiotics.

Conclusions: MIURA program has positively influenced on decreasing antibiotic prescription in our health area, as shows the decrease in DHD during the interventions.

Key words: Antibiotics. Off-Label Use. Drug utilization.

(*) Este trabajo ha sido parcialmente financiado por el FIS (PI C03/14 y PI 031624).

Los autores han declarado no tener ningún conflicto de intereses. 


\section{INTRODUCCIÓN}

Las enfermedades infecciosas y su tratamiento son un motivo de constante y creciente preocupación en materia de política sanitaria, por la conocida morbilidad, mortalidad y coste que ocasionan. La monitorización e información dinámica sobre los procesos infecciosos y el consumo de antimicrobianos son de gran relevancia para la gestión clínica, pues permiten observar la evolución de un conjunto de características de la población asistida y permite la toma de decisiones sobre actividades de control ${ }^{1}$. Sin embargo, los métodos de vigilancia del uso de antimicrobianos todavía no se han estandarizado suficientemente de forma integrada en las distintas áreas sanitarias, dada la habitual separación en la gestión de los distintos niveles asistenciales.

La instauración de estrategias de uso racional de antibióticos es una prioridad de salud a nivel mundial ${ }^{2}$. La Unión Europea, en las recomendaciones del Consejo de 15 de noviembre de 2001 sobre la utilización de agentes antimicrobianos en la medicina humana, ya recomendaba la existencia de estrategias específicas coordinadas intersectoriales para el uso adecuado de estos medicamentos, a fin de contener, entre otros, el progresivo incremento de bacterias resistentes $^{3}$. Por todo ello se precisa de un enfoque pragmático, basado en el diseño de estrategias integradas, orientado a lograr una utilización óptima de los antimicrobianos.

En el último cuarto de siglo se han observado en España ciertas mejoras en el proceso de racionalización del tratamiento de procesos infecciosos, con un menor consumo extrahospitalario de antibióticos parenterales, una disminución de las asociaciones de antinfecciosos y un mayor control de los antibacterianos orales de amplio espectro, como consecuencia de la voz de alarma dada por las OMS y las sociedades científicas acerca del problema de las resistencias bacterianas ${ }^{4}$. Sin embargo, este proceso de racionalización no ha alcanzado todavía su nivel adecuado, ya que España se encuentra entre los países de la Unión Europea con un mayor consumo de antibióticos ${ }^{5}$.

En la actualidad no está consensuado ni existe un método de referencia para mejorar el uso eficiente de antibióticos en un área salud. Está demostrado que las acciones voluntaristas llevadas a cabo por personal poco experimentado y las intervenciones puntuales que no se consolidan en el tiempo terminan en fracaso ${ }^{6}$. Por este motivo, para que los programas de uso racional de medicamentos resulten exitosos deben combinar varios tipos de intervenciones, y ser conducidos por personal multidisciplinar, especializado y en condiciones de liderazgo. Como la finalidad de estas estrategias es su consolidación en el tiempo, el método a aplicar debe adaptarse a las características de la institución y al personal al cual va dirigido, con el objeto de que el programa resulte aceptable y se asegure la mejor calidad de la atención médica al menor costo.

En enero de 2004 comenzó a desarrollarse, en el Departamento de Salud 11 (Alzira) de la Comunidad Valenciana, el programa MIURA (Modelo Integrado para el Uso Racional de Antimicrobianos), en el cual, mediante diversos tipos de intervenciones dirigidas a médicos, farmacéuticos y pacientes, se pretendía mejorar el uso de los antinfecciosos en el área de salud. El objetivo del presente trabajo ha sido evaluar la repercusión de la implantación de este programa mediante el análisis de la evolución de los antibióticos dispensados con receta.

\section{SUJETOS Y MÉTODOS}

Ámbito del estudio. El estudio se realizó en el Departamento de Salud de Alzira, cuyo hospital de referencia es el Hospital Universitario de La Ribera (282 camas). Se encuentra situado en la localidad Alzira y atiende a la población de la comarca de La Ribera 
(1.011 $\mathrm{Km}^{2}$ de superficie) en la provincia de Valencia. El área dependiente del Hospital engloba a algo más de 250.000 habitantes (con aproximadamente 40.000 niños menores de 14 años), lo que representa un $6 \%$ de la población de la Comunidad Valenciana. La densidad de población del área es de unos 200 habitantes $/ \mathrm{Km}^{2}$ y casi el $80 \%$ vive en municipios de más de 10.000 habitantes.

Proyecto MIURA e intervenciones sanitarias realizadas. El MIURA es un programa de múltiples intervenciones que pretende fomentar el uso racional de antimicrobianos en un área de salud. Para ello, un grupo multidisciplinar de especialistas del Hospital, representando a diversos Servicios (Microbiología, Farmacia Hospitalaria, Medicina Interna, Pediatría, Atención Primaria y Farmacia Comunitaria), planificó un cronograma de intervenciones de tipo educativo y no restrictivas, que se iniciaron en enero de 2004 y finalizaron en diciembre de 2006.

Las intervenciones fueron programadas y realizadas trimestralmente, e iban dirigidas a personal sanitario (médicos de Atención Primaria, Especializada y personal de enfermería; [nivel I]), oficinas de farmacia; [nivel II]), y la comunidad (pacientes; [nivel III]). Brevemente, entre ellas caben destacar: jornadas científicas dirigidas a personal sanitario, carteles informativos para la comunidad, artículos de información sobre resistencias bacterianas y consumo de antibióticos en el Departamento de Salud, cuñas publicitarias en radio y televisión locales, folletos tipo cómic sobre el uso racional de antimicrobianos, charlas en centros de salud y en centros de amas de casa y jubilados, tarjetas plastificadas (dirigidas a médicos de Atención Primaria) indicando los agentes etiológicos más probables y el tratamiento empírico de elección en los principales procesos infecciosos, etiquetas adhesivas (dirigidas a oficinas de farmacia) para indicar la posología y la duración del tratamiento antibiótico a cada paciente, diseño de una aplicación informática encargada de la información del consumo de antibióticos y resistencias bacterianas en el Área, y divulgación de material didáctico relacionado con el buen uso de los antinfecciosos (tabla 1).

Diseño y mediciones. Se realizó un estudio observacional, descriptivo y comparativo sobre la evolución de la dispensación de antibióticos con receta durante los periodos 2000-03 (pre-MIURA), 2004-06 (MIURA) y 2007-08 (post-MIURA) en el Departamento de Salud 11 de la Comunidad Valenciana.

La información se obtuvo a través de la aplicación informática GAIA de la Dirección General de Prestación Farmacéutica de la Conselleria de Sanitat (Generalitat Valenciana $)^{7}$. Este sistema recoge información sobre los medicamentos facturados por las oficinas de farmacia a través de las recetas oficiales de la Seguridad Social.

Los antibióticos estudiados fueron los incluidos en el grupo J01 (antibacterianos para uso sistémico) del sistema de clasificación Anatomical Therapeutic Chemical Classification (ATC) de la OMS. No se han estudiado, por tanto, los antibióticos de uso no sistémico, antivirales, antimicóticos, ni antimicobacterianos.

Como unidad técnica de medida se empleó la DHD (Dosis diaria definida/1.000 Habitantes/Día). Se obtuvieron los datos de DHD de antibióticos para farmacia gratuita (DHD-pensionista), farmacia activa (DHD-activa) y DHD-estandarizada (DHD estadísticamente corregida en base a la estructura poblacional, por situación de activo o pensionista, del Departamento de Salud). También se obtuvieron datos DHD de antibióticos por subgrupos terapéuticos y principios activos.

Los resultados obtenidos se analizaron para los periodos 2000-2008. Se analizó el impacto del MIURA durante el periodo de 


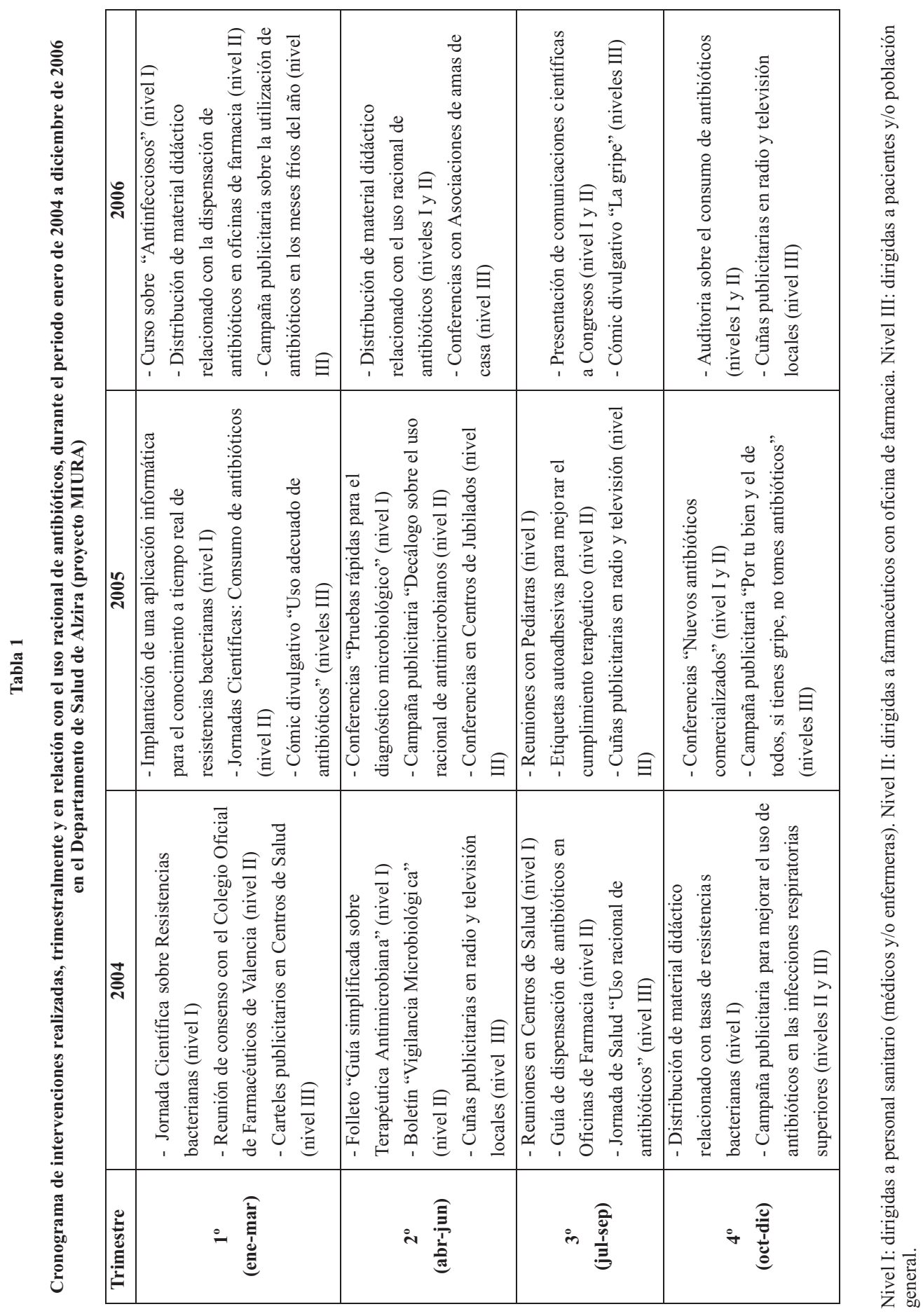


su implantación, mediante comparación con los datos obtenidos para los periodos 200003 y 2007-08. Para el análisis estadístico se empleó el programa SPSS v12.0. Se utilizó la $t$ de Student para comparar el número de DHD obtenidas. En todos los cálculos estadísticos se consideró un error de tipo I $(\chi)$ igual a 0.05 .

\section{RESULTADOS}

Los datos de antibióticos (DHD) dispensados con receta durante el periodo 2000-08 en el Departamento de Salud de Alzira se muestran en la tabla 2. Desde la instauración del proyecto MIURA se observa una tendencia a disminuir el número de DHD entre los años 2004 y 2006. Así, presentó un pico máximo de 27,19 DHD en el año 2003, disminuyendo progresivamente hasta 23,17 DHD en el año 2006, lo que representa una reducción global y significativa del $15 \%$ desde que comenzó el MIURA $(\mathrm{p}<0,05)$. Como era de esperar, se detectaron importantes diferencias entre pensionistas y población activa, observando que para los primeros los valores de DHD son más del doble que para los segundos $(41,17$ frente a $17,39$ para el año 2006; $\mathrm{p}<0,05)$. En el periodo post-MIURA (2007-08) se observó un incremento en las DHD, en comparación con el dato del 2006, de 1,54 DHD.

Tal como se muestra en la tabla 3, el grupo terapéutico más prescrito durante todos los años de estudio fue el de las penicilinas (con una DHD media anual de 11,47), seguido de macrólidos (con 4,42 DHD) y cefalosporinas (con 4,14 DHD). Entre los años 2003 (antes de la instauración del MIURA) y 2006 (al final del MIURA), se observó una disminución estadísticamente significativa $(\mathrm{p}<0.05)$ en los valores de DHD para los grupos terapéuticos de macrólidos $(5,73$ frente a $2,98)$ y de cefalosporinas $(4,62$ frente a 3,76). También existió una discreta disminución significativa, para el grupo de las quinolonas, mientras que los grupos de penicilinas y de otros antibióticos no presentaron

Tabla 2

Dispensación de antibióticos con receta, en Dosis diaria definida/1.000 Habitantes/Día (DHD), en el Departamento de Salud 11 (Alzira) de la Comunidad Valenciana durante los años 2000-2008

\begin{tabular}{|l|c|c|c|}
\hline Año & DHD-pensionista & DHD-activa & DHD-estandarizada \\
\hline 2000 & 51,96 & 18,53 & 26,89 \\
\hline 2001 & 48,29 & 17,10 & 25,06 \\
\hline 2002 & 49,44 & 18,20 & 26,12 \\
\hline 2003 & 50,88 & 19,24 & 27,19 \\
\hline $2004^{*}$ & 50,07 & 18,15 & 26,15 \\
\hline $2005^{*}$ & 48,17 & 18,56 & 25,79 \\
\hline $2006^{*}$ & 41,17 & 17,39 & 23,17 \\
\hline 2007 & 42,84 & 18,20 & 24,21 \\
\hline 2008 & 42,71 & 18,29 & 24,24 \\
\hline
\end{tabular}

* Comienzo y desarrollo del proyecto MIURA. 
Tabla 3

Número de dosis diaria definida por 1.000 habitantes y día (DHD) de grupos antibióticos en el Departamento de Salud de Alzira de la Comunidad Valenciana durante los años 2000-2008

\begin{tabular}{|l|r|r|r|r|r|r|r|r|r|r|}
\hline $\begin{array}{c}\text { Grupo } \\
\text { Terapéutico }\end{array}$ & $\mathbf{2 0 0 0}$ & $\mathbf{2 0 0 1}$ & $\mathbf{2 0 0 2}$ & $\mathbf{2 0 0 3}$ & $\mathbf{2 0 0 4} *$ & $\mathbf{2 0 0 5} *$ & $\mathbf{2 0 0 6} *$ & $\mathbf{2 0 0 7}$ & $\mathbf{2 0 0 8}$ & $\begin{array}{c}\text { Media anual } \\
\text { (desv. estandar) }\end{array}$ \\
\hline $\begin{array}{l}\text { B-lactámicos } \\
\text { penicilinas }\end{array}$ & 11,57 & 10,35 & 10,75 & 11,45 & 11,09 & 11,95 & 11,46 & 12,26 & 12,38 & $11,47(0,67)$ \\
\hline $\begin{array}{l}\text { B-lactámicos: } \\
\text { cefalosporinas }\end{array}$ & 4,46 & 4,11 & 4,16 & 4,62 & 4,12 & 4,21 & 3,76 & 4,00 & 3,85 & $4,14(0,27)$ \\
\hline Macrólidos & 5,62 & 5,08 & 5,71 & 5,73 & 4,73 & 4,34 & 2,98 & 2,83 & 2,73 & $4,42(1,27)$ \\
\hline Quinolonas & 3,27 & 3,46 & 3,43 & 3,38 & 3,23 & 3,33 & 3,08 & 3,23 & 3,40 & $3,31(0,12)$ \\
\hline Otros & 1,97 & 2,05 & 2,06 & 2,02 & 2,99 & 1,98 & 1,89 & 1,88 & 1,87 & $2,08(0,35)$ \\
\hline Total & 26,89 & 25,06 & 26,12 & 27,19 & 26,15 & 25,79 & 23,17 & 24,20 & 24,23 & $25,42(1,35)$ \\
\hline
\end{tabular}

* Comienzo y desarrollo del proyecto MIURA.

Tabla 4

Evolución de la dispensación, en Dosis diaria definida/1.000 Habitantes/Día, de los principales antibióticos en el Departamento de Salud de Alzira de la Comunidad Valenciana durante los años 2000-2008

\begin{tabular}{|l|r|c|c|c|c|c|c|c|c|c|}
\hline $\begin{array}{l}\text { Principio } \\
\text { Activo }\end{array}$ & $\mathbf{2 0 0 0}$ & $\mathbf{2 0 0 1}$ & $\mathbf{2 0 0 2}$ & $\mathbf{2 0 0 3}$ & $\mathbf{2 0 0 4} *$ & $\mathbf{2 0 0 5} *$ & $\mathbf{2 0 0 6} *$ & $\mathbf{2 0 0 7}$ & $\mathbf{2 0 0 8}$ & $\begin{array}{c}\text { Media anual } \\
\text { (desv. estandar) }\end{array}$ \\
\hline $\begin{array}{l}\text { Amoxicilina/ } \\
\text { Clavulánico }\end{array}$ & 5,88 & 5,56 & 6,15 & 6,98 & 7,40 & 7,93 & 7,76 & 8,36 & 8,32 & $7,15(1,06)$ \\
\hline Amoxicilina & 5,49 & 4,58 & 4,42 & 4,29 & 3,54 & 3,88 & 3,53 & 3,74 & 3,92 & $4,15(0,62)$ \\
\hline Claritromicina & 2,70 & 2,72 & 3,19 & 3,28 & 2,65 & 2,38 & 1,49 & 3,04 & 3,15 & $2,73(0,55)$ \\
\hline Cefuroxima & 2,81 & 2,73 & 2,84 & 3,36 & 2,98 & 3,05 & 2,83 & 1,45 & 1,34 & $2,60(0,71)$ \\
\hline Ciprofloxacino & 1,71 & 1,72 & 1,69 & 1,66 & 1,51 & 1,43 & 1,38 & 1,29 & 1,31 & $1,52(0,18)$ \\
\hline Azitromicina & 1,21 & 1,17 & 1,36 & 1,47 & 1,31 & 1,24 & 0,49 & 0,95 & 1,03 & $1,19(0,18)$ \\
\hline Doxiciclina & 0,72 & 0,84 & 0,91 & 0,84 & 0,88 & 0,74 & 0,68 & 0,53 & 0,51 & $0,74(0,15)$ \\
\hline Norfloxacino & 1,04 & 0,99 & 0,91 & 0,81 & 0,72 & 0,62 & 0,51 & 0,47 & 0,43 & $0,72(0,23)$ \\
\hline Cefixima & 0,72 & 0,72 & 0,69 & 0,66 & 0,64 & 0,63 & 0,51 & 0,58 & 0,42 & $0,62(0,10)$ \\
\hline Levofloxacino & 0,15 & 0,29 & 0,26 & 0,23 & 0,38 & 0,65 & 0,69 & 0,94 & 1,24 & $0,54(0,37)$ \\
\hline Cotrimoxazol & 0,55 & 0,54 & 0,54 & 0,52 & 0,43 & 0,42 & 0,42 & 0,39 & 0,36 & $0,46(0,07)$ \\
\hline Moxifloxacino & 0,27 & 0,38 & 0,49 & 0,63 & 0,51 & 0,52 & 0,42 & 0,48 & 0,38 & $0,45(0,10)$ \\
\hline Total & 23,25 & 22,24 & 23,45 & 24,73 & 22,95 & 23,49 & 21,16 & 22,22 & 22,41 & 22,88 \\
\hline
\end{tabular}

* Comienzo y desarrollo del proyecto MIURA. 


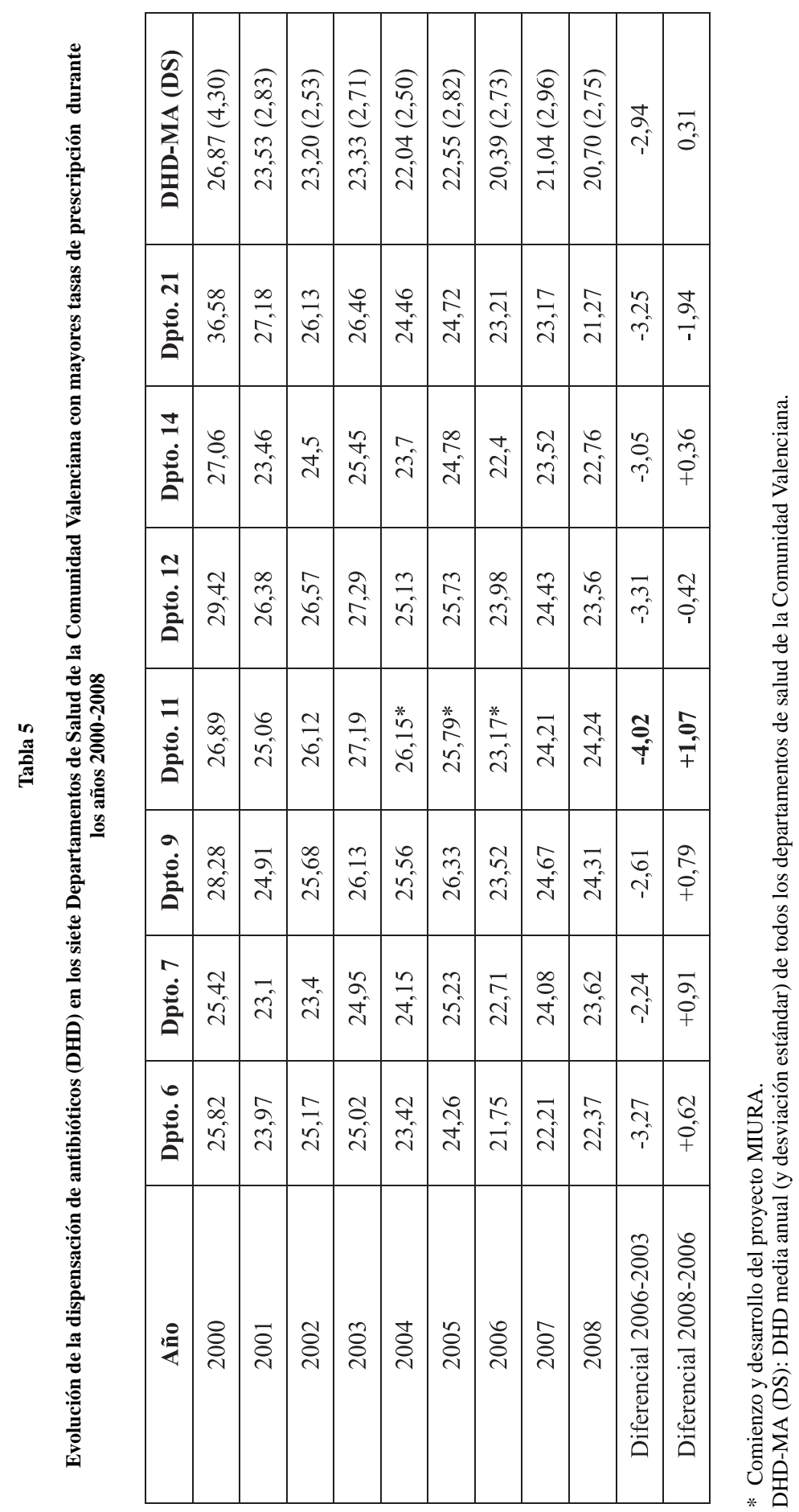


gran variabilidad en los datos. En el periodo post-MIURA, y en comparación con el año 2006, se observó un ligero incremento (no significativo) en la mayoría de los grupos estudiados, con excepción de los grupos de macrólidos y de otros antibióticos.

El análisis por principios activos (tabla 4), mostró que el más consumido de los $\beta$-lactámicos fue la asociación amoxicilina-clavulánico, seguido de amoxilina, cefuroxima y cefixima. Dentro del grupo de los macrólidos, el más utilizado es claritromicina, seguido de azitromicina. Penicilina y eritromicina fueron escasamente empleados (datos no mostrados). Desde la instauración de intervenciones sanitarias a través del MIURA, se detectó una disminución significativa en la dispensación de claritromicina (3,28 DHD en el año 2003 frente a 1,49 DHD en 2006; p<0.05), mientras que para amoxicilina, cefuroxima, cefixima, azitromicina, ciprofloxacino y norfloxacino se observó una disminución estadísticamente no significativa. Otros antibióticos, como amoxicilina-clavulánico y levofloxacino, experimentaron un incremento en la dispensación (incluso durante el periodo de instauración del MIURA). En el periodo postMIURA el análisis por principios activos mostró variabilidad respecto a los datos del 2006, aunque globalmente se observó un incremento en la dispensación de los antibióticos estudiados, ya que al comparar el dato de DHD total entre los años 2006 y 2008 se observó un incremento de 1,25 DHD.

En la tabla 5 se muestra la evolución de antibióticos de los siete Departamentos de Salud de la Comunidad Valenciana con mayores niveles de dispensación. Aunque se observa una tendencia general en la disminución de antibióticos en todos los Departamentos, se puede apreciar que desde la instauración del proyecto MIURA, el Departamento 11 fue el que mayor grado de disminución presentó (con un diferencial entre 2003-2006 de -4,02 DHD). En el periodo
2007-08, cuando ya no se realizaban intervenciones, se observó un progresivo incremento en la dispensación de antibióticos en nuestro Departamento, con un diferencial entre 2008-2006 de +1,07 DHD.

\section{DISCUSIÓN}

El desarrollo de un proyecto como el MIURA parece incidir en la disminución del consumo de antibióticos, ya que tras su implantación se detectó una reducción global del $15 \%$ en los tres años de duración del proyecto. Aunque las modificaciones en las tendencias de utilización de antimicrobianos son multifactoriales, el programa de intervenciones formativas ha podido contribuir significativamente al cambio de estas tendencias. Al no tener un grupo de control, es difícil atribuir completamente los resultados obtenidos a las intervenciones realizadas aunque, al comparar los datos de dispensación de antibióticos con los de otras áreas de salud similar (tabla 5), se observa una marcada y favorable tendencia en el Departamento de salud 11 (donde se implementó el MIURA).

La reducción del consumo de antibióticos durante el periodo 2004-06 se produjo sobre todo por la reducción en el colectivo de los pensionistas (tabla 1). Varias razones pueden explicar esta reducción. Por un lado, este grupo poblacional es el que mayor cantidad de antibióticos utiliza (siendo en consecuencia el más susceptible de disminuir), y por otro lado, gran parte de las intervenciones de tipo III realizadas por el MIURA fueron dirigidas a este colectivo.

Los españoles presentan un grado de información sobre los antibióticos y las resistencias bacterianas muy bajo, y éste se ha relacionado con la mala utilización de los $\operatorname{mismos}^{8,9,10}$. Ello condiciona un problema de salud pública de primer orden, que requiere una respuesta global al mismo ${ }^{11}$. La administración sanitaria, las sociedades 
científicas y los propios centros sanitarios a nivel local deben de esforzarse en informar sobre las consecuencias del mal uso de los antibióticos $^{12}$.

Los antimicrobianos representan la décima parte del mercado farmacéutico, y son los productos de mayor consumo tras los analgésicos y los de mayor gasto tras los antihipertensivos. En España, en el año 2002 se dispensaban menos de 20 DHD, cifra muy por debajo de las 31 DHD de mediados de la década de 1970 , pero que todavía sitúa a España entre los países de más elevado consumo, sobre todo si se compara con el Reino Unido, Alemania o los países nórdicos ${ }^{13}$. Durante los años 2000-2003, los datos en el área de La Ribera no estaban en consonancia con la media nacional, ya que el consumo de antimicrobianos correspondiente al año 2003 era de 27,19 DHD. Estos datos no estaban justificados, por lo que se promovió la instauración de estrategias, a través del MIURA, que probablemente han contribuido en una reducción global en 4 DHD en la dispensación oficial de antibióticos.

Los estudios de consumo describen las cantidades de los medicamentos dispensados. Son útiles como sistemas de alerta, para detectar desviaciones de consumo, comparar zonas de salud o médicos, y aproximar la calidad del medicamento prescrito. Aunque la utilización de datos basados en las recetas prescritas con cargo a la Seguridad Social es un método aceptado ${ }^{14}$, presenta la limitación de no contemplar el consumo asociado a la libre administración farmacéutica ni a la práctica privada. Un reciente estudio ${ }^{15}$ pone de manifiesto que la estimación del consumo de antibióticos en España varía fuertemente según el método de medición, ya que el consumo estimado a través de datos de reembolso (receta médica) es sustancialmente menor (aproximadamente un 30\%) que a través de datos de ventas. Estas diferencias son especialmente significativas para los principales antibióticos prescritos (amoxicilina/clavulánico, amoxicilina, claritromicina y cefuroxi- ma), con las implicaciones que puede tener para su vigilancia.

Otra consideración adicional a tener en cuenta es que la cuantía de dispensaciones y la toma de medicamentos no pueden considerarse indicadores estrictamente sinónimos. El objeto de este estudio ha sido medir la cantidad de antibióticos dispensados y no la calidad en la indicación de los mismos. Los estudios prescripción-indicación valoran el grado de adecuación de la prescripción, y se suelen aplicar a medicamentos nuevos, caros, de difícil manejo, o muy prescritos, para los que existen pautas claras de prescripción. Su principal limitación práctica es que son muy demandantes de información, que no siempre existe o está disponible. Adicionalmente requieren bases de datos fiables de historias clínicas y prescripciones, así como protocolos o guías de adecuación inequívocas.

El número de dosis diarias definidas (DDD) por 1000 habitantes y día (DHD) es una unidad cuantitativa de medida internacionalmente reconocida para los estudios de consumo de medicamentos en la población ${ }^{16}$, ya que permite valorar tendencias y resulta especialmente útil para comparar entre distintas áreas de salud. Esta unidad salva las dificultades de comparación entre envases que contienen diferentes cantidades del principio activo.

Se han propuesto diversas intervenciones para mejorar las prácticas de consumo de antibióticos en la atención ambulatoria ${ }^{17}$. La mayoría de ellas van dirigidas a abordar el abuso de antibióticos para las infecciones virales y la elección o duración del tratamiento para las infecciones bacterianas más frecuentes (faringitis estreptocócica, infecciones urinarias, otitis media aguda). El uso de materiales educativos impresos o auditoría y retroalimentación (feedback) por sí solos, no parecen producir cambios significativos en la prescripción. Las reuniones educativas interactivas parecen ser más efi- 
caces que las clases didácticas. Las visitas de extensión educativa y los recordatorios médicos producen resultados variados. Las intervenciones basadas en pacientes, en particular el uso de prescripciones diferidas para infecciones en las que los antibióticos no se indicaron de inmediato, redujeron eficazmente el uso de antimicrobianos por parte de los pacientes y no dieron lugar a exceso de morbilidad. Las intervenciones multifacéticas que combinaron educación para los médicos, los pacientes y el público en una variedad de sitios y formatos, fueron las más exitosas en la reducción de la prescripción inadecuada de antibióticos. Por este motivo, se decidió utilizar este formato para el diseño del MIURA, aunque en este trabajo no se ha pretendido evaluar el impacto relativo que han podido tener cada una de ellas, ni cuál ha sido la influencia de las intervenciones realizadas en otros aspectos como las tasas de resistencia bacteriana, la incidencia de reacciones adversas o el grado de cumplimiento terapéutico.

El uso irracional del medicamento es un problema de salud multifactorial que precisa la participación de todos los implicados, y especialmente el apoyo institucional. En el entorno hospitalario, el grado de intervención es más favorable, ya que las distintas Comisiones Hospitalarias (Infecciosas, Farmacia, Calidad, etc) establecen criterios de selección y utilización de antibióticos, los difunden y realizan un seguimiento para conseguir el máximo cumplimiento. Sin embargo, en Atención Primaria la situación es más compleja debido a la diversidad de factores que influyen negativamente sobre el correcto uso de los antibióticos, siendo necesarias estrategias múltiples que combinen distintas intervenciones ${ }^{18}$.

Actualmente no existen en España mecanismos intersectoriales (atención primaria, atención especializada, oficinas de farmacia o farmaindústria) ni estrategias coordinadas que se apliquen en áreas sanitarias amplias. En la Comunidad Valenciana existe un Plan de Armonización de las medidas y objetivos en materia de uso racional de Medicamentos (PARME) que pretende implicar a todos los agentes que intervienen en la cadena terapéutica, pero que requiere de adaptaciones locales, tipo MIURA, a cada área de salud.

Existe la posibilidad de actuar sobre las conductas de uso de antibióticos y mejorarlas, pero resulta indispensable considerar el contexto en forma global y de manera integrada, involucrando a médicos, farmacéuticos y pacientes, y manteniendo las intervenciones periódicamente ${ }^{19}$. Sólo con perseverancia es posible cambiar los hábitos inadecuados de prescripción, dispensación y consumo, de allí que un cronograma anual de intervenciones, realizado por profesionales o Comisiones con experiencia en el tema de antibióticos, ajustado a las peculiaridades de cada área de salud y ejecutado de forma constante a lo largo de los años, sea en nuestra experiencia un forma eficaz de realizar una correcta política de antibióticos.

\section{AGRADECIMIENTOS}

A la Conselleria de Sanitat de la Comunidad Valenciana por facilitar el acceso a la información y utilizar su sistema informático. A los Dres. Ángel Aguilar (Hospital Universitario de La Ribera, Alzira) y José Campos (Instituto de Salud Carlos III) por su inestimable ayuda en la revisión crítica del manuscrito. Al Dr Vicente Palop (Hospital Universitario de La Ribera, Alzira) por su apoyo incondicional durante toda la duración del proyecto MIURA.

\section{BIBLIOGRAFÍA}

1. Tonna AP, Stewart D, West B, Gould I, McCaig D. Antimicrobial optimisation in secondary care: the pharmacist as part of a multidisciplinary antimicrobial programme-a literature review. Int J Antimicrob Agents. 2008;31:511-7. 
2. Campos J, Baquero F. Antibiotic resistance: what to do now?. Med Clin (Barc). 2002;119:656-8.

3. Diario Oficial de las Comunidades Europeas. Recomendación del Consejo 2002/77/CE relativa a la utilización prudente de los agentes antimicrobianos en la medicina humana. DOCE núm L034 de $5 / 2 / 2002$.

4. Lázaro E, Madurga M, de Abajo FJ. Trends in antibiotic consumption in Spain, 1985-2000. Med Clin (Barc). 2002;118:561-8

5. Goossens H, Ferech M, Coenen S, Stephens P; European Surveillance of Antimicrobial Consumption Project Group. Comparison of outpatient systemic antibacterial use in 2004 in the United States and 27 European countries. Clin Infect Dis. 2007;44:1091-5.

6. Thomson O'Brien MA, Oxman AD, Haynes RB, Davis DA, Freemantle N, Harvey EL. Local opinion leaders: effects on professional practice and health care outcomes. Cochrane Database Syst Rev. 2007;1:CD000125.

7. Trillo J, Villalba P, De la Torre P. GAIA: Gestor Integral de Prestación Farmacéutica. Boletín Farmacoterapéutico Valenciano 2002;1:51-54. [citado 01-02-2010]. Disponible en http://www.san.gva. es/val/prof/dgf/farmacia/pdf/11jul.pdf

8. Palop Larrea V, Martínez-Mir I. Documento de consenso sobre la utilización de antibióticos en atención primaria. Aten Primaria. 2006;38:137-8.

9. Branthwaite A, Pecheré JC. Pan-European Survey of Patients. Attitudes to antibiotics and antibiotic use. J Inst Med Research. 1996; 24:229-38.

10. Clemente Lirola E, Millaina G, Moreno Luna E, Vacas Ruiz AL. Sobre la cultura antibiótica de la población. Aten Primaria. 2000;26:136.

11. Smith RD, Coast J. Antimicrobial resistance: a glo- bal response. Bulletin of the World Health Organization. 2002;80:126-33.

12. Palop V, Melchor A, Martínez-Mir I. Reflexiones sobre la utilización de antibióticos en atención primaria. Aten Primaria. 2003;32:42-7.

13. Lazaro E, de Abajo FJ. Uso de antibióticos en España. Agencia Española de Medicamentos y Productos Sanitarios (AEMPS) y Dirección General de Farmacia y Productos Sanitarios (DGFPS), 2007. [citado 01-02-2010]. Disponible en http://www.agemed.es/profHumana/observatorio/docs/uso-antibioticos-oct07.pdf

14. Bremón AR, Ruiz-Tovar M, Gorricho BP, de Torres PD, Rodríguez RL. Non-hospital consumption of antibiotics in Spain: 1987-1997. J Antimicrob Chemother. 2000;45:395-400.

15. Campos J, Ferech M, Lázaro E, de Abajo F, Oteo J, Stephens P, Goossens H. Surveillance of outpatient antibiotic consumption in Spain according to sales data and reimbursement data. J Antimicrob Chemother. 2007;60:698-701.

16. WHO Collaborating Centre for Drug Statistics Methodology: Anatomical Therapeutical Chemical (ATC) Classification Index. Including defined daily dosis (DDDs) for plain substances. Oslo: WHO CCDSM; 1995.

17. Arnold SR, Straus SE. Interventions to improve antibiotic prescribing practices in ambulatory care. Cochrane Database Syst Rev. 2005;4:CD003539.

18. Ruiz Contreras J. Estrategias para neutralizar el impacto de las resistencias bacterianas. An Esp Pediatr. 2002; 56 (Suppl. 1): 59-64.

19. Von Gunten V, Reymond JP, Beney J. Clinical and economic outcomes of pharmaceutical services related to antibiotic use: a literature review. Pharm World Sci. 2007;29:146-63. 